EPJ Web of Conferences 59, 08012 (2013)

DOI: $10.1051 /$ epjconf/20135908012

(C) Owned by the authors, published by EDP Sciences, 2013

\title{
Analysis of electromagnetic pulse (EMP) measurements in the National Ignition Facility's target bay and chamber
}

\author{
C.G. Brown Jr. ${ }^{a}$, T.J. Clancy, D.C. Eder, W. Ferguson and A.L. Throop \\ Lawrence Livermore National Laboratory, Livermore, CA 94550, USA
}

\begin{abstract}
From May 2009 to the present we have recorded electromagnetic pulse (EMP) strength and spectrum $(100 \mathrm{MHz}-5 \mathrm{GHz})$ in the target bay and chamber of the National Ignition Facility (NIF). The dependence of EMP strength and frequency spectrum on target type and laser energy is discussed. The largest EMP measured was for relatively low-energy, short-pulse (100 ps) flat targets.
\end{abstract}

\section{INTRODUCTION}

Protection of sensitive diagnostics at laser facilities requires information on EMP strength and frequency spectrum. See [1-6] for previous work on EMP in laser facilities. The National Ignition Facility (NIF) is the world's largest laser with up to $1.8 \mathrm{MJ}$ of $3 \omega$ laser energy. Currently NIF operates with pulse durations from approximately 0.1 to $30 \mathrm{~ns}$. An advanced diagnostic system planned for NIF, the Advanced Radiographic Capability (ARC), requires shorter pulse durations in the 1 to $10 \mathrm{ps}$ range. Based on results presented here and data from our work at the short-pulse Titan laser facility [5, 6], we expect the EMP on NIF to increase significantly during ARC operation. We fielded similar EMP sensors in NIF's target bay and chamber that we used earlier on Titan. We start with some background on NIF and explain the benefits of ongoing monitoring of EMP. We follow with a brief description of the measurement system on NIF. We conclude with very interesting results on the energy scaling of EMP as a function of target type.

\section{EMP AT NIF}

Given that NIF has almost two orders of magnitude higher laser energy than any other laser system, there was significant concern with regard to EMP mitigation in the design of the diagnostics. For this work, it was assumed that the EMP would be in the $100 \mathrm{MHz}$ range with shielding specifications extending to $1 \mathrm{GHz}$ to be conservative. Because NIF was designed to field igniting fusion targets, which produce on order of $10^{19}$ neutrons, the majority of sensitive diagnostic components, e.g., oscilloscopes, are placed in one of four diagnostic mezzanines located behind 2-m thick shield walls. Diagnostics components placed inside the chamber are placed in well-shielded enclosures. However, it is not possible to completely shield any diagnostic and still obtain the needed data. In addition, our work on the Titan short-pulse laser showed that the EMP can extend out to higher frequencies (5 GHz and above) $[5,6]$, which is higher than NIF's original design specification for shielding. This provided motivation to measure EMP on NIF prior to full energy operation to measure energy scaling and to also determine if some targets produced larger EMP. Results of that study are given here. There are benefits to having

\footnotetext{
ae-mail: brown207@llnl.gov
}

This is an Open Access article distributed under the terms of the Creative Commons Attribution License 2.0, which permits unrestricted use, distribution, and reproduction in any medium, provided the original work is properly cited. 


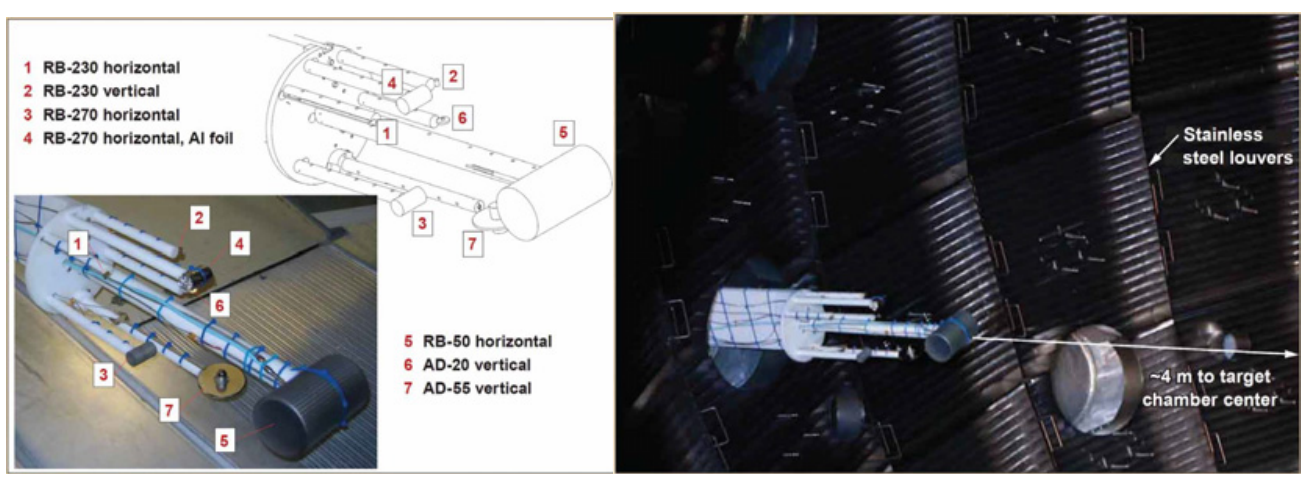

Figure 1. Target chamber sensors.

an ongoing measurement system. If data loss or instrument damage is observed, comparison of present EMP measurements with past EMP measurements aids in determining whether the cause was EMP fields. In addition, since the source and properties of the EMP on NIF are not fully understood, ongoing measurements can be used for early trend detection. If EMP fields are observed to rise based on a trend (e.g., increasing yield, shorter pulses, certain types of targets or diagnostic features, etc.), mitigation steps can be taken before deleterious effects occur.

\section{MEASUREMENT SYSTEM}

We have fielded a suite of B-dot (loop) and D-dot (monopole) sensors [7] in the target bay and chamber. Figure 1 displays the sensors used in the target chamber. Target bay sensors currently operating (not shown) consist of the same types of sensors and are mounted on movable stands. All of the sensors are commercial, free-field B- and D-dot sensors from Prodyn [8] that operate in a differential mode, which allows reduction in common-mode noise by subtracting the differential signals using baluns [7]. For brevity we call the target chamber sensors "internal" and the target bay sensors "external".

The internal B-dot sensors consist of one RB-50 ( $\sim 300 \mathrm{MHz})$; two RB-270 ( $\sim 1 \mathrm{GHz})$, where one sensor is enclosed in an EMI shield to allow a noise-only measurement; and two RB-230 ( $\sim 5 \mathrm{GHz})$. All of the internal B-dot sensors are oriented horizontally, except for one of the RB-230 sensors, which is oriented vertically. Additionally, there are two internal D-dot sensors. One is an AD-55 ( $\sim 2 \mathrm{GHz})$, and the other is an AD-20 $(\sim 10 \mathrm{GHz})$. Both internal D-dot sensors are oriented vertically. All of the internal B- and D-dot sensors are mounted on dielectric supports (see Fig. 1). The signals from the sensors are routed using dual coaxial cables (with the shields bonded along their lengths), through nonisolated vacuum feedthroughs, into a flange box in the target bay, where they are subtracted using baluns, which typically provide over $30 \mathrm{~dB}$ common-mode rejection up to a few $\mathrm{GHz}$. The RB-50 uses a type "F" balun, which has a bandwidth of $200 \mathrm{kHz}-3.5 \mathrm{GHz}$. All of the other internal sensors use type "G" baluns, with a bandwidth of $250 \mathrm{kHz}-10 \mathrm{GHz}$. Additionally, the output of the baluns is filtered with appropriate low-pass filters to control aliasing and to limit the operation of each sensor to its differentiating mode. The external sensors are one RB-50 B-dot; one RB-270 B-dot; two RB-230 B-dots; and two AD-55 D-dots. The cables from the baluns of both the internal and external sensors are routed to a rack in the diagnostic mezzanine, where high-bandwidth oscilloscopes (10 and 20 GS/s Tektronix 6604 and 6604 B) acquire the signals. Off-line data processing deconvolves the B-field data from the raw oscilloscope measurements and regularizes the result by applying a $125 \mathrm{MHz}$ digital high-pass filter. While the measurement system is well shielded against electromagnetic fields, the internal sensors, particularly the AD-55 and the RB-50, are possibly affected by x rays. We are currently assessing the effects on the measurements and possible mitigations. We introduced the digital high-pass filter in the data processing in part to reduce what we think are x-ray effects on the data. 



$$
\text { Titan, } 132 \mathrm{~J}, 2 \text { ps pulse, } 1 \mathrm{~mm} \text { x } 1 \mathrm{~mm} \text { x } 12 \mathrm{um} \mathrm{Ag} \text { foil }
$$
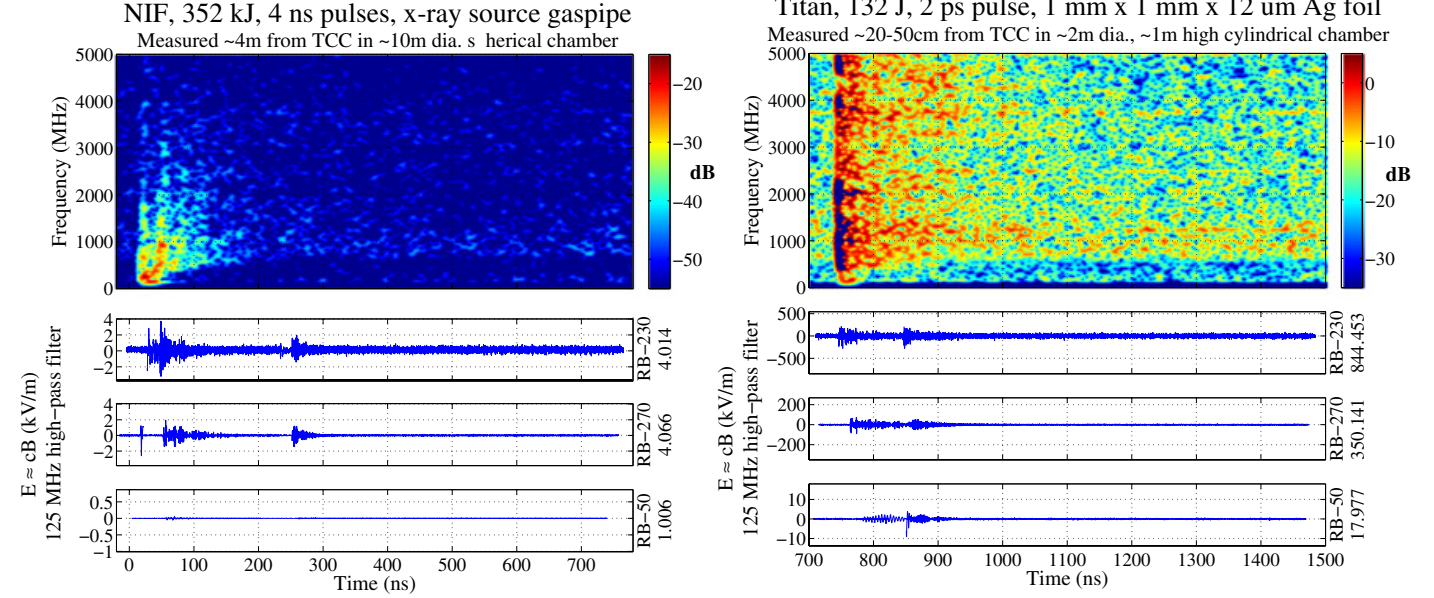

Figure 2. E-field traces and composite spectrograms for various laser energies and target types.

\section{RESULTS AND DISCUSSION}

For the purposes of this paper, we have selected data from 15 NIF shots to focus on. The shots represent a wide range of laser parameters (pulse duration and laser energy) and target geometries. In Fig. 2, we give E-field traces (derived from the retrieved B-fields using a plane wave relationship, which is an approximation) for each of the three B-dot sensors and a composite spectrogram for three NIF shots: one from the National Ignition Campaign (NIC) (upper left), one from the timing and pointing efforts using a flat target (upper right), and one from the X-Ray Source Application (XRSA) campaign using a gaspipe (lower left). The composite spectrograms are formed by cross-fading the individual spectrograms of each of the B-dot sensors along the frequency axis, where the cross-fade transition regions are around the upper limits of the frequency range of the RB-50 and RB-270 sensors. In addition, one data set from our work on the Titan short-pulse laser is included for comparison (lower right). See the final paragraph in this section for a discussion of scaling. The most dramatic result is the very low EMP for the NIC target at $\sim 1 \mathrm{MJ}$ of laser energy. The flat target with $2 \mathrm{~kJ}$ of laser energy and a $0.1 \mathrm{~ns}$ pulse has the largest EMP for the three NIF shots but still well below the Titan shot for $132 \mathrm{~J}$ and 2 ps pulse, although the Titan measurement is much closer to the target. 


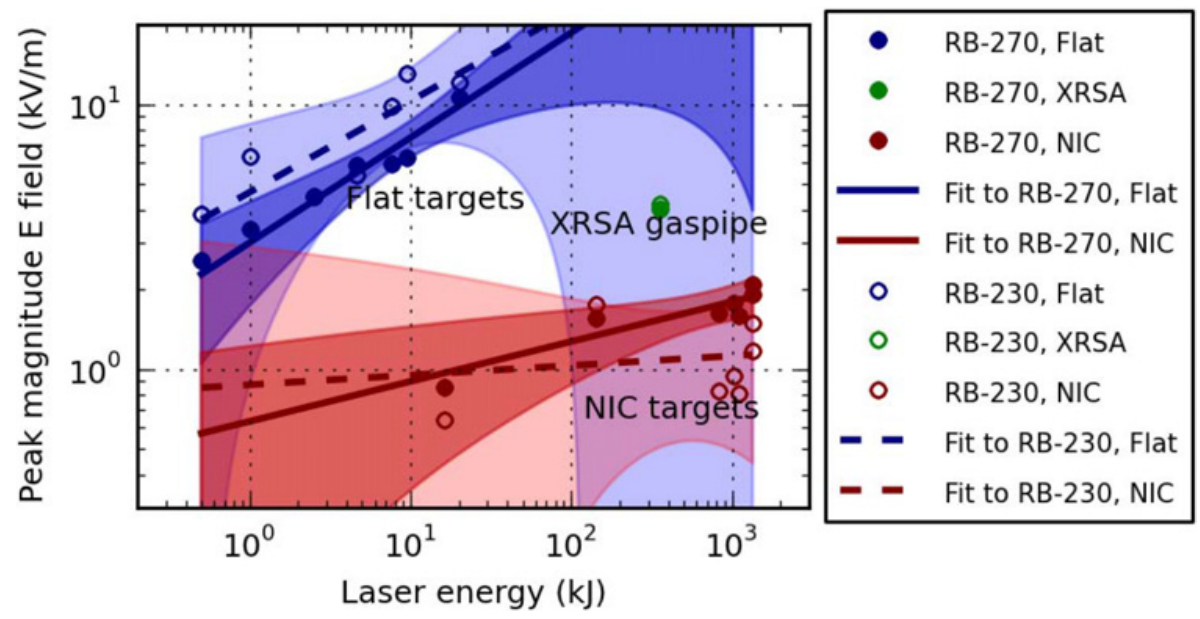

Figure 3. Peak E-field magnitude versus energy and target type with least-squares fits and corresponding $95 \%$ simultaneous confidence bands.

The spectrograms also show that the EMP for the NIC campaign has relatively little high frequency content. This is also seen in Fig. 3 where we display peak E-field magnitude (using data from the high frequency RB-230 and the medium frequency RB-270 B-dot sensors) versus energy and target type, grouping into flat, XRSA, and NIC targets. For the NIC shots, the RB-230 gives lower field values as compared to the RB-270 except for one shot. In contrast for flat targets, the higher frequency RB-230 generally gives higher field values than the lower frequency RB-270. In the XRSA experiment, the two sensors give comparable values. Least-squares fits of the peak E field values versus energy and their corresponding 95\% simultaneous confidence bands are plotted in Fig. 3.

A major result of this study is the relatively low (few $\mathrm{kV} / \mathrm{m}$ ) EMP associated with NIC targets. The EMP for the low-energy timing and pointing flat targets is generally order of magnitude higher. The XRSA experiment falls between these in EMP strength. Including the Titan data, we find that the EMP extends out to higher frequencies as the laser pulse becomes shorter in addition to the increase in strength. This is important for mitigation associated with ARC operation on NIF. Since measurements in the NIF chamber are made $\sim 4 \mathrm{~m}$ from Target Chamber Center (TCC), EMP fields could be higher nearer to TCC. For example if $1 / \mathrm{r}^{2}$ spherical spreading loss of power is assumed, $\sim 1 \mathrm{~m}$ from TCC fields could be $\sim 4 \mathrm{x}$ higher than the fields measured at $\sim 4 \mathrm{~m}$ for TCC. Scaling methods other than spherical spreading loss of power, such as treating the source as a small dipole or loop antenna, could be employed and might yield more accurate results.

\section{References}

[1] M. Mead et al., Rev. Sci. Instrum. 75(10), 4225 (2004)

[2] J. Raimbourg, Rev. Sci. Instrum. 75(10), 4234 (2004)

[3] C. Stoeckl et al., Rev. Sci. Instrum. 77, 10F506 (2006)

[4] J.L. Bourgade et al., Rev. Sci. Instrum. 79(10), 10F301 (2008)

[5] C.G. Brown Jr., A. Throop, D. Eder, J. Kimbrough, J. Phys.: Conf. Ser. 112032025 (2008)

[6] C.G. Brown Jr. et al., J. Phys.: Conf. Ser. 244032001 (2010)

[7] W.R. Edgel, http://www.prodyntech.com/download/appnotes/PAN\%20606.pdf. Accessed May 2012

[8] Prodyn Technologies, http://prodyntech.com. Accessed May 2012. 